${ }^{\odot}$ Entomologica Fennica. 29.III.1993

\title{
Hemiptera of Iraq. II. Cydnidae, Thaumastellidae, Pentatomidae, Stenocephalidae, Coreidae, Alydidae, Rhopalidae, and Pyrrhocoridae
}

\section{Rauno E. Linnavuori}

Linnavuori, R. E. 1993: Hemiptera of Iraq. II. Cydnidae, Thaumastellidae, Pentatomidae, Stenocephalidae, Coreidae, Alydidae, Rhopalidae, and Pyrrhocoridae. - Entomol. Fennica 4:37-56.

The article contains a list of the known records of the Cydnidae, Thaumastellidae, Pentatomidae, Stenocephalidae, Coreidae, Alydidae, Rhopalidae, and Pyrrhocoridae of Iraq and Kuwait. Notes on the biology and distributional data are also included. New synonymy: Aethus sahlbergi (Reuter) $=A$. hispidulus (Klug). Lectotype is designated for Aethus sahlbergi (Reuter).

Rauno E. Linnavuori, Somersoja, SF-21220 Raisio 22, Finland

Surprisingly few records have hitherto been published on the Hemipteran fauna of Iraq and $\mathrm{Ku}$ wait. The present paper is primarily based on my own field work in 1979-1981, when I was working in the Ministry of Agriculture, Abu Ghraib, in Iraq. The collection of this institute was also revised. The new species detected were published by me separately in 1984 .

\section{Cydnidae}

\section{Sehirinae}

Sehirus robustus Horváth, 1895

Material: Duhok, 1 ex, 12.VIII.1972, A1-Qadi, in AG (= the Plant Protection Department, Ministry of Agriculture, Abu Ghraib, Baghdad).

Distribution: Anatolian, known from Turkey and Caucasia.
Canthophorus melanopterus (Herrich-Schäffer, 1835)

Material: Ana, 1 ex, 25.VI.1966; Rawa, 3 exx, 24.VI.1966, in AG.

Distribution: Holomediterranean.

Exosehirus validus (Jakovlev, 1877)

N Iraq (Hoberlandt 1961:200).

Distribution: Irano-Turanian.

Exosehirus sargon Linnavuori, 1984

Exosehirus sargon Linnavuori 1984:2.

Material: Irbil, near Salahuddin, male holotype, 1314.VI.1980, Linnavuori, in coll. Linnavuori.

Distribution: Endemic.

Crocistethus waltlianus (Fieber, 1836)

Material: Kuysunjag-Irbil, 1 ex, 12.IV.1980, Linnavuori.

Distribution: Holomediterranean. 
Ochetostethus opacus (Scholtz, 1847)

Material: near Darbandikhan, 1 ex, 13.V.1980; Shaykh Addi, 1 ex, 11.V.1981, Linnavuori.

A revision of the genus in Kerzhner 1976:3343.

Distribution: Holomediterranean with a wide range in Central and southern parts of North Europe.

\section{Cydninae}

Cydnus aterrimus (Forster, 1771)

Material: Mosul, many exx, 28.VIII.1980; Al Qosh, 1 ex, 25.VIII.1980, Linnavuori.

Distribution: Holomediterranean, extending into Central Europe and the Oriental Region.

Amaurocoris curtus (Brullé, 1838)

Material: Najaf-Shabakah, 2 exx, 23.VI.1981, Linnavuori.

Distribution: Eremian.

\section{Amaurocoris orbicularis Jakovlev, 1885}

Material: Nukhayb, 5 exx, 2.V.1980; Nukhayb - km 160, 1 ex, 3.V.1980; Ukhaydir-Nukhayb, 1 ex, 2.V.1980, Linnavuori.

Distribution: Eremian.

\section{Aethus hispidulus (Klug, 1845)}

Cydnus hispidulus Klug 1845:43.

Aethus hispidulus Signoret 1881:431.

Aethus setosus Walker 1867:39 (Linnavuori 1993:75).

Cydnus sahlbergi Reuter 1900:222, syn. n.

Types: Iraq, Euphrates, male lectotype of setosus, designated by Linnavuori, in the British Museum; Greece, Corfu, female syntype of sahlbergi, here designated as the lectotype, U. Sahlberg, in Mus. St. Petersburg.

Taxonomy of the species will be treated by me elsewhere (Linnavuori 1993). An examination of a syntype of Cydnus sahlbergi Reuter in Mus. St. Petersburg revealed that the species is a synonym of $A$. hispidulus.

Distribution: Apparently Pontomediterranean, recorded from Greece, Cyprus, Syria, Israel, Egypt, Arabia, and Iraq.
Aethus rugosus (Jakovlev, 1874)

Material: Abu Ghraib, 5 exx, 29.V.1965, 6.VI.1966, 22.VI.1967, in AG; near Safwan, 2 exx, 25.VI.1980, Linnavuori.

Distribution: Irano-Turanian.

Aethus pilosulus (Klug, 1845)

Material: Many exx from Abu Ghraib, 6.VI.1956, 15.XI.1965, 1-8.IX.1966, in AG; Baghdad, IX.1979, IVVII.1980; Al Haditha, 13.VIII.1979; Tharthar lake, 23.VIII.1979, 2.VI.1980, Linnavuori.

Distribution: Holomediterranean.

Byrsinocoris nigroscutellatus Montandon, 1900

Material: Arabia: Oman Desert, 1 ex, in coll. Linnavuori.

Distribution: Eremian; previously known from Algeria. The first record from The Arabian Peninsula.

Macroscytus brunneus (Fabricius, 1803)

Material: Numerous exx from Abu Ghraib; Darband, 24.VIII.1970, J. Abdulla; Duhok, 12.VIII.1972, I. Naser; Mosul, 24.VI.1973, in AG; Baghdad, IV-VII.1980; Sarsang, 26-28.VIII.1980, Linnavuori.

Distribution: Widely distributed within the Holomediterranean subregion and the Oriental and Ethiopian Regions. Recorded from Iraq (Stichel 1962:775).

Geotomus elongatus (Herrich-Schäffer, 1840)

Material: Arbil, 1 ex, 21.I.1968, J. Sultan, in AG.

Distribution: Holomediterranean, extending into Central Europe, the Middle East and Turkestan.

Geotomus pygmaeus (Dallas, 1851)

Material: Numerous exx from Abu Ghraib, 30.V.1953, 5.XI.1965, 15.IX.1966, 8.VII and 28.X.1967, in AG; Ain al Tamar, 17.VII.1980; Amara, 23-24.X.1979; Samawa, 21.X.1979, Linnavuori.

Distribution: The Oriental Region, extending into Iraq, the eastern parts of Saudi Arabia and the Jordan Valley in Israel. 
Geotomus intrusus Wagner, 1953

Material: Abu Ghraib, several exx, 30.III.1953, 6.VI.1956, in AG; Baghdad, 3 exx, IV-VIII.1980, Linnavuori.

Distribution: Eremian.

\section{Thaumastellidae}

\section{Thaumastella aradoides Horváth, 1896}

Material: Baghdad, 2 exx, IX.1979, Linnavuori; also recorded from Baghdad in Seidenstücker (1964:274).

Distribution: Eremian.

\section{Pentatomidae}

\section{Scutellerinae}

\section{Odontoscelis litura (Fabricius, 1775)}

Material: Al Qosh, ơ neotypus, ơ paraneotypus, designated by Göllner-Scheiding, 10.V.1981, Linnavuori, in coll. Linnavuori.

Göllner-Scheiding (1986) published a revision of the genus Odontoscelis Laporte de Castelnau and proved that $O$. litura is a valid species and not a form of $O$. fuliginosa as previously assumed.

Biology: The specimens were found on ground among vegetation in a brook valley at the foot of a hill.

Distribution: Syrio-Anatolian. Recorded from Egypt, Syria, Iraq, Cyprus and Turkey.

\section{Odontoscelis dorsalis (Fabricius, 1803)}

Material: near Bawa Nur, 1 ex, 10.VI.1980; A1 Qosh, 1 ex, 10.V.1981; As Salman-Shabakah, several exx, 4.IV.1981; Waqisah, several exx, 10.IV.1981, Linnavuori.

Biology: On ground among vegetation in sandy habitats.

Distribution: Holomediterranean.

\section{Alphocoris halophilus Linnavuori, 1984}

Types: Tharthar Lake, $0^{7}$ holotype, several paratypes, 20.X.1980; Al Hillah, 1 paratype, 21.IX.1979; Sawa Lake, 2 paratypes, 19.IV.1980, Linnavuori, in coll. Linnavuori.
Material: Abu Ghraib, 1 ex, 8.IV.1958; S. Rashed; Diwaniya, 1 ex, 11.IX.1954, S. Alyasiri, in AG.

Description in Linnavuori 1984:3-4.

Biology: On Chenopodiaceae halophytes in saline habitats.

Distribution: Endemic.

\section{Odontotarsus robustus Jakovlev, 1883}

Material: several exx from Aqra, 12.V.1981; Al Qosh, 10.V.1981; Sawarah Tuka, 28.VIII.1980, Linnavuori.

Biology: Swept from vegetation in hilly steppes.

Distribution: Holomediterranean.

Odontotarsus rufescens Fieber, 1861

Recorded from Shaqlawa (Hoberlandt 1953: 378).

Distribution: Pontomediterranean.

Odontotarsus purpureolineatus (Rossi, 1790)

Material: Aqra, 2 exx, 12.V.1981; Al Qosh, 2 exx, 10.V.1981; Sawarah Tuka, 1 ex, 28.VIII.1980; Zawita, 1 ex, 26.VIII.1980; Linnavuori; Bismil, 2 exx, 31.VII.1963, Y. Baysed.

Biology: On vegetation in hilly steppes of northern Iraq.

Distribution: Holomediterranean.

Odontotarsus impictus Jakovlev, 1886

Recorded from Shaqlawa (Hoberlandt 1953: 378).

Distribution: Irano-Turanian.

Odontotarsus armiger Kiritshenko, 1914

Material: Al Hadr, 2 exx, 6-7.V.1981; Al Qosh, 1 ex, 25.VIII.1980; Khan Ruhabah, 1 ex, 6.IV.1981; Shaykh Addy, 1 exx, 11.V.1981, Linnavuori.

Biology: In steppes of northern Iraq.

Distribution: Irano-Turanian. Known from the Sinai Peninsula, Jordan and Turkestan.

Odontotarsus angustatus Jakovlev, 1883

Material: Darbandikhan, 1 ex, 21.V.1980; Sawarah Tuka, 1 ex, 28.VIII.1980, Linnavuori; Jamjamal, 1 ex, 21.V.1980, R. T. Kasnaw. 
Previously recorded from Bishapur (Hoberlandt 1949:2).

Biology: In steppes of northern Iraq.

Distribution: Irano-Turanian, recorded from Iraq, Iran and Turkestan.

Psacasta cypria Puton, 1881

Material: Qora, 1 ex, 12.VI.1980, Linnavuori.

Taxonomy treated in Seidenstücker 1975: 265-266.

Biology: Swept from vegetation in a steppe in northern Iraq.

Distribution: Anatolian, recorded from Cyprus, Turkey and Iraq (Seidenstücker 1975:265266).

Eurygaster integriceps Puton, 1881

Material: Numerous exx from Gali Ali Beg, 13.VI.1980; Shaykh Addy, 11.V.1981; Linnavuori; Abu Ghraib, 2.VIII.1954, A. Mahmood; El Abbasiye, 29.III.1944, Y. Fethi; Biyara, 19.III.1944, Y. Yousuf; Dekon, 29.V.1951, A. Meymarian; Sulaymaniyah, 11.V.1951, Abiel, in AG.

Biology: In steppes and fields in northern and central Iraq.

Distribution: Pontomediterranean.

\section{Podopinae}

Tarisa camelus Reuter, 1901

Material: Sawa Lake, 1 ex, 21.X.1979, Linnavuori.

Biology: On Anabasis articulata in a saline sandy habitat.

Distribution: Eremian, recorded from Algeria, Egypt, Israel and Saudi Arabia.

\section{Tarisa subspinosa (Germar, 1839)}

Material: Numerous exx from Ain Al Tamar-Ramadi, 20.IV.1980; Amara, 23-24.X.1979; Ana, 10.IV.1981; AnaAl Qaim, 5.V.1980; Busayah-As Salman, 2.IV.1981; Hilla, 21.IX.1979; Kerbala-Ain Al Tamar, 20.X.1979; Khan Ruhabah, 6.IV.1981; Rashidiya, 8.VI.1981; Rawah-A1 Hadr, 6.V.1981; Nasiriyah-Abu Ghar, 15.IV.1980; Razazah Lake, 6.VI.1981; As Salman-Samawa, 6.III.1980; Sawa Lake, 21.X.1979; Tharthar Lake, 20.VIII.1979, Linnavuori.
Taxonomy treated in Linnavuori 1986:51-52.

Biology: In saline habitats on Anabasis articulata, Suaeda vermiculata and Zygophyllum. Hibernating specimens on Lycium barbarum.

Distribution: Eastern parts of the Eremian subregion. Recorded from Egypt, Saudi Arabia, Syria, Iraq, Kuwait, and Central Asia.

Tshingisella bella Kiritshenko, 1913

Material: Bawa Nur, 1 ex, 10.VI.1980; Shaykh Addy, 1 ex, 11.V.1981, Linnavuori.

Biology: Swept from vegetation in hilly steppes in northern Iraq.

Distribution: Irano-Turanian, recorded from Turkey, Syria and Central Asia.

Leprosoma inconspicuum Baerensprung, 1859

Material: near Salahuddin, 1 ex, 12.VI.1980, Linnavuori.

Biology: Swept from vegetation in a hilly steppe in northern Iraq.

Distribution: Holomediterranean.

Ventocoris horvathi (Puton, 1896)

Material: Al Qosh, 1 ex, 10.V.1981, Linnavuori.

Recorded from Shaqlawa (Hoberlandt 1953: 378).

Biology: Swept from vegetation in a hilly steppe.

Distribution: Syrio-Anatolian, recorded from Syria, Turkey, Iraq, and Iran.

Ventocoris obesus (Stål, 1865)

Material: Khan Ruhabah, 1 ex, 6.IV.1981; NajafShabakah, 1 ex, 23.VI.1981, Linnavuori.

Biology: On Zilla spinosa.

Distribution: Eremian, known from Algeria, Egypt, Israel, and Saudi Arabia.

Ventocoris achivus (Horváth, 1889)

Material: Al Qosh, 2 exx, 25.VIII.1980, Linnavuori.

Taxonomy treated in Josifov 1969:67-72.

Biology: Swept from vegetation in a hilly steppe in northern Iraq.

Distribution: Pontomediterranean. 
Ventocoris modestus (Jakovlev, 1880)

Material: Khan Ruhabah, 1 ex, 24.VI.1981, Linnavuori.

Biology: From vegetation in a sandy habitat.

Distribution: Eremian.

Ancyrosoma leucogrammes (Gmelin, 1789)

Material: many exx from Darbandikhan, 13.V.1980; Dukan, 12.VI.1980; Penjwin, 11.VI.1980; Al Qosh, 10.V.1981; Rashidiyah, 8.VI.1981; Salahuddin, 1314.VI.1980; Sawarah Tuka, 28.VIII.1980; Zawita, 26.VIII.1980; Sinjar, 7.V.1980; Sulaf, 27.VIII.1980, Linnavuori; Abu Ghraib, 29.III.1955, I. Kheiri; Khanken, 3.IV.1958, collector unknown; Khorsabad, 10.VI.1944, A. Meymarian, in AG.

Biology: Common on vegetation in steppes and gardens.

Distribution: Holomediterranean; recorded from Iraq (Stichel 1961:740).

\section{Tholagmus enkidu Linnavuori, 1984}

Material: Mosul, ơ holotype and 1 o paratype, 26.VII.1967, J. Alani; Al Qosh, 1 paratype, 10.VI.1981; Shayk Addi, 1 paratype, 11.V.1981, Linnavuori, in coll. Linnavuori.

Revision of the genus Tholagmus Stål in Linnavuori 1984:4-7.

Biology: In hilly steppes in NE Iraq.

Distribution: Endemic.

Graphosoma semipunctatum (Fabricius, 1775)

Material: Amadia, 3 exx, 21.VI.1968, Salih, in AG.

Distribution: Holomediterranean, extending into Central Europe, Central Asia and Afghanistan.

\section{Graphosoma lineatum italicum (Müller, 1766)}

Material: Sulaf, several exx, 27.VIII.1980, Linnavuori.

Biology: On Umbelliferae in a hilly steppe.

Distribution: Holomediterranean with a wide range into Central Europe and Central Asia.

\section{Graphosoma stali Horváth, 1881}

Material: Cony-Masy, 1 ex, 26.VI.1975, Irwad; Duhok, 3 exx, 26.V.1965, collector unknown, in AG.
Distribution: Syrio-Anatolian, recorded from Turkey, Syria, Iraq (Seidenstücker 1975:267), and Iran.

\section{Pentatominae}

Mecidea pallidissima Jensen-Haarup, 1922

Material: Baghdad, 1 ex, VI-VII.1980; Nukhaib, 1 ex, 2.V.1980, Linnavuori.

Biology: On grasses in sandy habitats.

Distribution: Eremian, distributed from West Africa to India.

Mecidea lindbergi Wagner, 1954

Material: Sinoni N of Sinjar, 1 ex, 8-9.VI.1981; Waqisah, 1 ex, 5.IV.1980, Linnavuori.

Biology: On grasses in sandy habitats.

Distribution: Eremian, distributed from the Cape Verde Islands to Iran. Recorded from Iraq by Stichel (1961:742).

Mustha spinulosa (Lefebvre, 1831)

Material: Dukan, 1 ex, 12.VI.1980; Salahuddin, 1 ex, 13-14.VI.1980; Sarsang, 1 ex, 26-28.VIII.1980; Sulaf, 1 ex, 27.VIII.1980, Linnavuori; Amadiah, 1 ex, 25.VII.1959, collector unknown; North Iraq, 2 exx, 23.VI.1965 and Shaqlawa, 11.VI.1940, A. Meymarian in AG.

Biology: On deciduous trees and shrubs such as Quercus in forests of NE Iraq.

Distribution: Pontomediterranean; recorded from Iraq in Stichel 1961:743.

\section{Apodiphus amygdali (Germar, 1817)}

Material: Many exx from Baghdad, 23.IX.1979, VVI.1981; Sulaf, 27.VII.1980; Zawita, 26.VIII.1980, Linnavuori; Aqrah, 24.VII.1968, J. Abdulla; Baghdad, 22.VIII.1965, 1.VIII.1969, A. Isa, M. Younis; Bakrajo, 4.VII.1972, A. Droity; Deken, 6.VIII.1951, A. Meymarian; Dora, 12.VII.1972, R. Alkhadil; Jadriye, 13.V.1944, M. Mubarek; Mosul, 13.II.1968; J. Abdulla; Shaqlawa, 15.VIII.1946, R. Hafidh; Tilgara, Arbil, 18.VI.1943, A. Meymarian; Zakho, 4.X.1961, collector unknown, in AG. Hinaidi near Baghdad (China 1938:428).

Biology: On deciduous trees and bushes in gardens and forests. In Iraq found on apple, apricot, mulberry, and plum.

Distribution: Pontomediterranean; recorded from Iraq in Stichel 1961:743. 


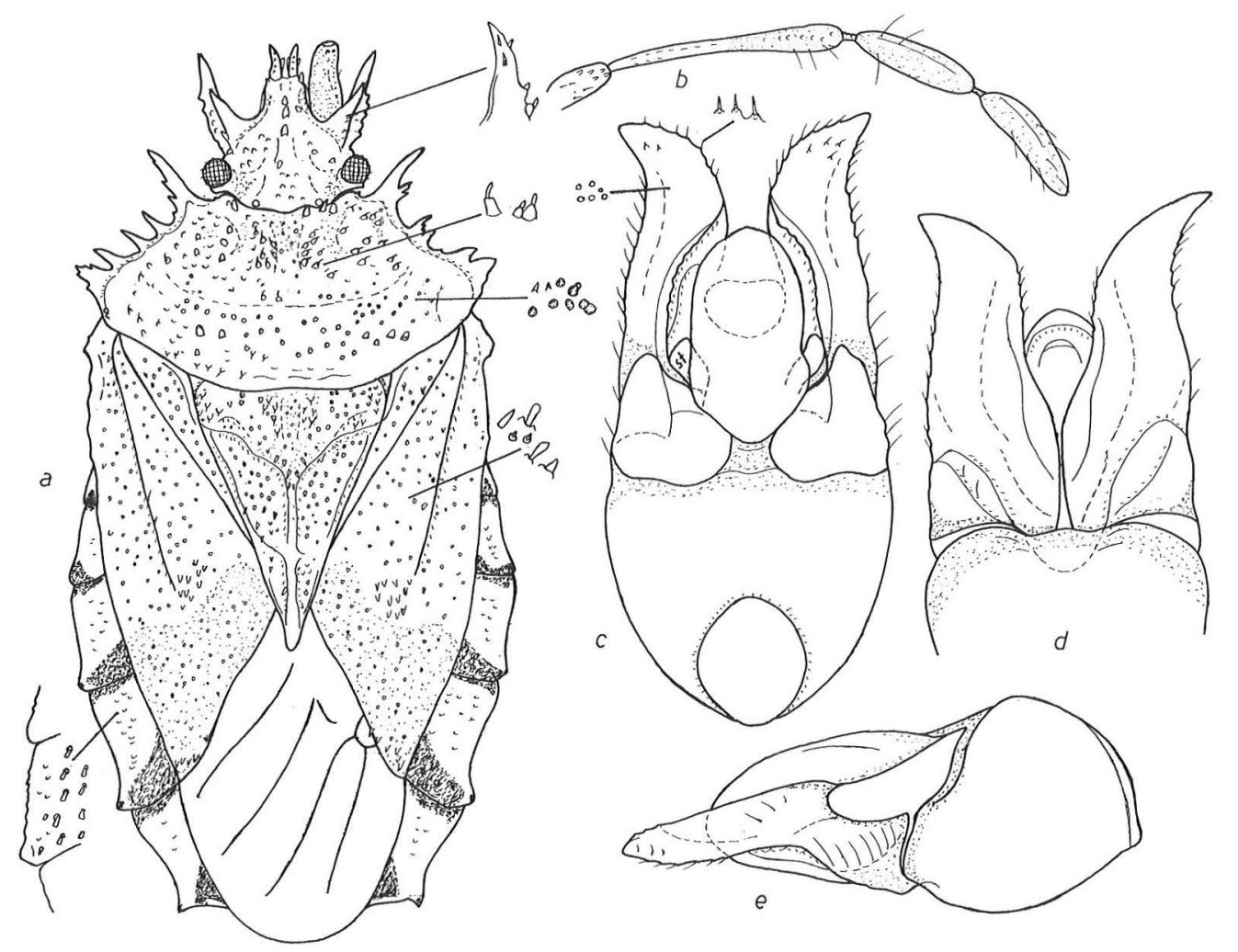

Fig. 1. Phricodus bessaci Villiers (macropterous male from Zubar). a: dorsal view; b: antenna; c-e: genital segment in dorsal, ventral and lateral view (st $=$ style).

Phricodus bessaci Villiers, 1959

Fig. 1

Material: Zubar, 1 ex, 14.IV.1980, Linnavuori.

Biology: In Saudi Arabia found on Heliotropium pterocarpum.

Distribution: Eremian, previously known from Senegal, Mauretania and Saudi Arabia (Linnavuori 1986:55).

\section{Sciocoris ochraceus Fieber, 1861}

Material: Abu Ghraib, 1 ex (det. Remane), 17.VI.1958, S. Rashed, in AG.

Distribution: Syrio-Anatolian.

\section{Sciocoris helferi Fieber, 1852}

Material: Darbandikhan, 3 exx, 13.V.1980; Dukan, 1 ex, 12.VI.1980; Khan Ruhabah, 1 ex, 24.VI.1981, Linnavuori.
Biology: On Labiatae, such as Thymus, in dry habitats.

Distribution: Holomediterranean, extending as far as Iran and Turkestan in the East.

Sciocoris safavii Hoberlandt, 1959

Mosul (Wagner 1965:128).

Distribution: Iranian, originally described from Iran.

Sciocoris capitatus Jakovlev, 1881

Material: Kuysanjag-Irbil, 2 exx, 12.VI.1980; Qora, 1 ex, 16.XI.1979, Linnavuori.

Biology: On Labiatae in hilly steppes.

Distribution: Irano-Turanian, recorded from Iran and Turkestan. 


\section{Sciocoris sahlbergi Wagner, 1952}

Material: Karbala-Ain Al Tamar, 3 exx, 20.IV.1980; As Salman-Takhadid, 2 exx, 17.IV.1980, Linnavuori.

Biology: On Labiatae in desert habitats.

Distribution: Eremian, known from Egypt, the Sudan, Israel, Saudi Arabia, and Turkey.

\section{Dyroderes umbraculatus (Fabricius, 1775)}

Material: Sulaf, 1 ex, 27.VIII.1980, Linnavuori.

Biology: On vegetation in dry hilly steppes.

Distribution: Holomediterranean, easternmost records from Turkey and Israel.

\section{Aelia Fabricius, 1803}

Taxonomy of the genus has been treated by China \& Lodos 1960 and Brown 1962.

\section{Aelia acuminata (Linnaeus, 1758)}

Material: Darbandikhan, 1 ex, 13.V.1980; Al Hadr, 1 ex, 6-7.V.1981; Rashidiyah, 3 exx, 8.VI.1981, Linnavuori; Abu Ghraib, numerous exx, 8.IV.1958, 9.VII.1958, S. Rashed, H. Abid, in AG.

Biology: On grasses in steppes and fields.

Distribution: Euro-Siberian.

\section{Aelia glebana Ferrari, 1874}

Material: Korasinnik, 2 exx, 6.VI.1944, A. Meymarian; Sinjar, 4 exx, 28.VI.1954, D. Ahmed, in AG.

Distribution: Holomediterranean with a wide range into Central Europe and Iran. Recorded from Iraq in China \& Lodos 1960:588.

\section{Aelia turanica Horváth, 1895}

Material: Salahuddin, 1 ex, 13-14.VI.1980; Sawarah Tuka, 4 exx, 28.VIII.1980, Linnavuori.

Pygofer in Fig. 2 d.

Biology: On grasses in hilly steppes.

Distribution: Irano-Turanian, recorded from Turkey, Iraq, Turkmenia, and Turkestan (Stichel 1961:748).
Aelia satunini Kiritshenko, 1929

Fig. 2a-c

Material: USSR, Aralych, Prov. Erivan, $10^{7}$ paratype, 11.V.1911, K. Satunin, in Mus. St. Petersburg.

Diagnosis: Distinguished from the related species by the immaculate legs (under surfaces of femora of the related species ornamented with a pair of dark subapical spots).

Description: Resembling A. turanica but smaller, length $7.5 \mathrm{~mm}$ (in turanica $8.0-9.5 \mathrm{~mm}$ ). Legs immaculate. - Small, body somewhat broader and more convex than in the related species. Head as broad as long, $0.6 \times$ as broad as basal width of pronotum, strongly sloping ventrad; ocular index 5.6. Under surface of head in lateral view with a shallow insinuation between the bluntly prominent apex of gena and buccula, the latter broadening caudad. Proportions between antennal joints 9:12:14:21:26 (diatone 53, basal width of pronotum 90 units). Pronotum twice as broad as long in middle, puncturing a little sparser than in turanica. Scutellum $1.17 \times$ as long as broad, somewhat broader than in the related species. Vein along claval suture on corium not elevated, less distinctly marked than in turanica. - Male genitalia: Pygofer (Fig. 2c) as in A. turanica but ventral margin in ventral view a little more strongly insinuated.

Distribution: Apparently Caspian. Also recorded from Turkey and Iraq (Shaqlawa, Hoberlandt 1953:378).

Aelia syriaca Horváth, 1903

Recorded from Amadiya, Korasinnik, Mosul, Sinjar, and Tel Kaif (Brown 1962:140).

Distribution: Syrio-Anatolian, also known from Syria and Turkey.

\section{Aelia albovittata Fieber, 1868}

Material: Kani Massi, 1 ex, 28.VI.1975, Rustam, in AG; Sarsang, 1 ex, 26-28.VIII.1980, Linnavuori.

Biology: On grasses in a hilly steppe.

Distribution: Syrio-Anatolian, recorded from Syria and Turkey. 


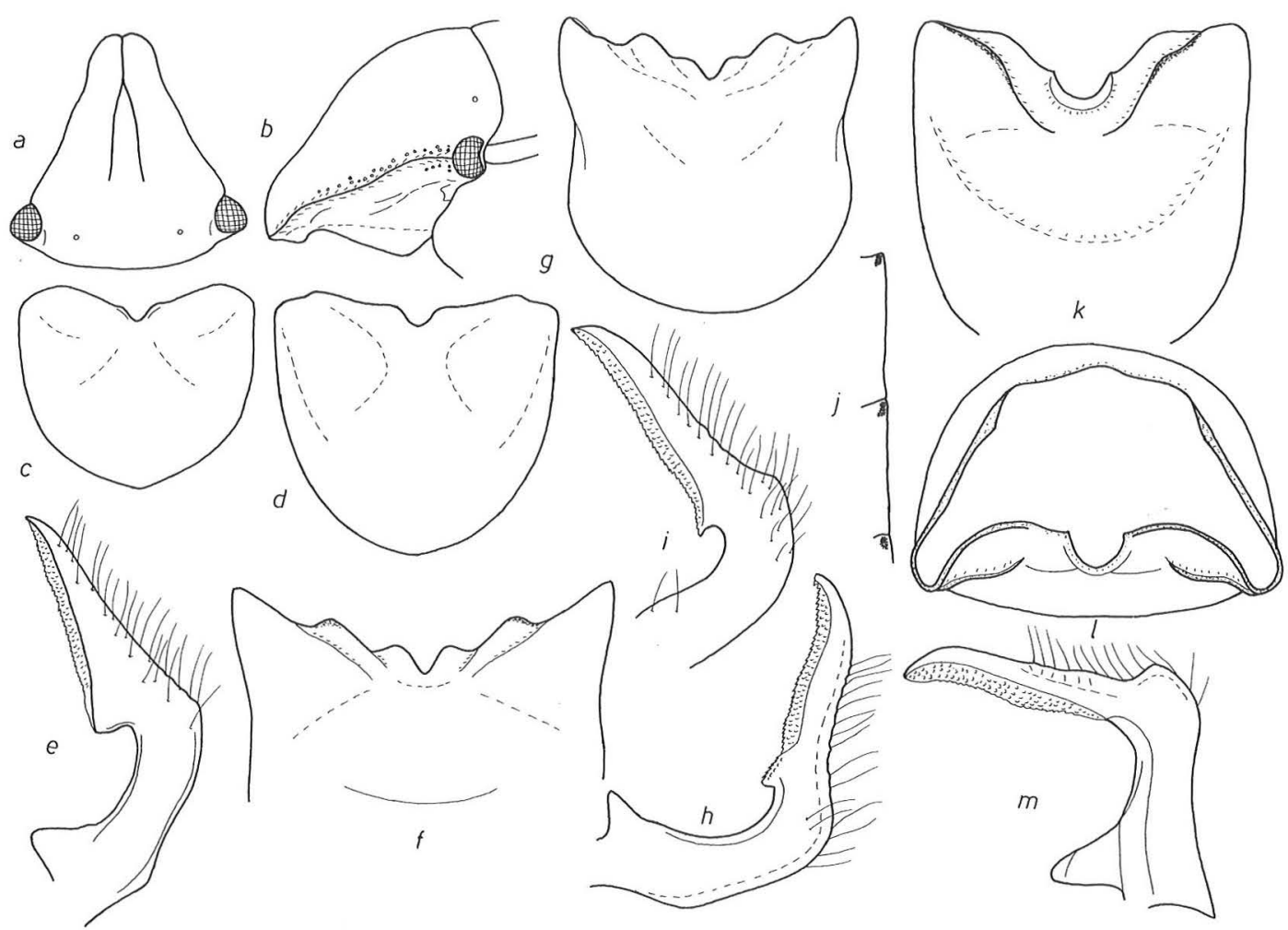

Fig. 2. Aelia satunini Kiritshenko. a-b: male head in dorsal and in lateral view; c: pygofer, caudal view. - A. turanica Horvath (ex from Sawara Tuka). d: pygofer, caudal view. - Holcostethus vernalis (Wolff) (ex from Raisio, Finland). e: style; f: pygofer, ventral view. - H. strictus (Fabricius). g: pygofer, ventral view; h-i: style (exx from Rashidiya and Qora). - H. congenitus Puchkov (ex from Sinoni). j: paratergites; $k-l$ : pygofer in ventral and in caudolateral view; m: style.

\section{Aeliomorpha sheanensis Linnavuori, 1965}

Material: Samawah, 5 exx, 21.X.1979, Linnavuori.

Biology: On Cyperaceae in a moist place in a garden.

Distribution: Previously known only from Israel.

\section{Neottiglossa bifida (Costa, 1847)}

Material: Aqrah, 1 ex, 12.V.1981; Brozah, 1 ex, 15.V.1981, Linnavuori.

Biology: Swept from grasses in hilly steppes.

Distribution: Holomediterranean.

\section{Eysarcoris ventralis (Westwood, 1837)}

Material: Numerous exx from Aqrah, 29.VIII.1980; Baghdad, VI-VII.1980; Halabja, 11.VI.1980; Al Kabaish, 27-28.VI.1980; Karbala-Ain Al Tamar, 20.X.1979; Mosul, 28.VIII.1980; Muhammadi, 8.X.1979; Penjwin, 11.VI.1980; Rashidiyah, 8.VI.1981; Sinjar, 14.XI.1979; Tharthar Lake, 25.VIII.1979, Linnavuori; Ana, 23.II.1955; Abu Ghraib, 6.VI.1955, Mounit; Basra, 25-29.VI.1980, Haifa'a M.Jalal, in AG.

Biology: On vegetation in gardens, fields and fresh meadows.

Distribution: Holomediterranean extending into the Ethiopian and Oriental Regions. 
Stagonomus amoenus (Brullé, 1832)

Material: Al Qosh, 1 ex, 10.V.1981, Linnavuori.

Biology: Swept from Labiatae in a hilly steppe.

Distribution: Holomediterranean, recorded from Iraq (Stichel 1961:750).

\section{Stagonomus bipunctatus (Linnaeus, 1758)}

Material: Zafatakia, 1 ex, 1.IX.1956, Ghalib, in coll. Linnavuori.

Distribution: Holomediterranean.

Stagonomus devius Seidenstücker, 1965

Material: Al Qosh, 2 exx, 10.V.1981, Linnvuori.

Biology: Swept from herbs in a hilly steppe.

Distribution: Syrio-Anatolian, recorded from Turkey, Israel.

\section{Gomphocranum christophi Jakovlev, 1877}

Material: Al Qosh, 3 exx, 10.V.1981, Linnavuori.

Taxonomy of the genus Gomphocranum Jakovlev treated in Linnavuori 1984:7-9.

Biology: Like the other species on shrubs on slopes of mountain valleys.

Distribution: Iranian, previously recorded from Iran and Israel.

\section{Gomphocranum adad Linnavuori, 1984}

Material: near Penjwin, ơ holotype, 11.VI.1980, Linnavuori.

Biology: On Capparis on a stony slope of a mountain valley.

Distribution: Endemic.

\section{Gomphocranum anu Linnavuori, 1984}

Material: Al Qosh, ơ holotype, 1 paratype, 17.XI.1979, 1 paratype, 24.VIII.1980; near Aqra, 1 paratype, 29.VIII.1980, Linnavuori.

Biology: On shrubs on slopes of mountain valleys.

Distribution: Endemic.

Staria lunata (Hahn, 1835)

Material: Shaykh Addi, 1 ex, 15.XI.1979, Linnavuori.

Biology: On Labiatae in a dry hilly steppe.

Distribution: Holomediterranean.
Holcostethus vernalis (Wolff, 1804)

Material: recorded from Baghdad (China 1934:429) and Shaqlawa (Hoberlandt 1953:380).

Pygofer and style illustrated in Fig. 2e-f.

Distribution: Euro-Siberian.

Holcostethus strictus (Fabricius, 1803)

Material: near Penjwin, 1 ex, 11.VI.1980; Qora, 4 exx, 16.XI.1979; Rashidiyah, 3 exx, 8.VI.1981; near Sawarah Tuka, 1 ex, 28.VIII.1980; Sulaf, 1 ex, 27.VIII.1980, Linnavuori.

Pygofer and style in Fig. $2 \mathrm{~g}-\mathrm{i}$.

Biology: On herbs in mountain steppes and gardens.

Distribution: Holomediterranean.

Holcostethus congenitus Puchkov, 1965

Material: near Darbandikhan, 1 ex, 13.V.1980; Khorsabad, 1 ex, 15.XI.1979; Sinoni, 1 ex, 9.V.1981, Linnavuori.

Pattern of connexivum in Fig. 2j. Pygofer and style in Figs. 2k-m, 3a.

Biology: On herbs in hilly steppes. At Khorsabad found on Olea.

Distribution: Irano-Turanian.

Palomena mursili Linnavuori, 1984

Material: Sulaf, ơ holotype and several paratypes, 27.VIII.1980, Linnavuori.

Description in Linnavuori 1984:9-10.

Biology: On deciduous trees in a dense wood in valley of a mountain brook.

Distribution: Iranian; also known from Iran.

Agatharchus tritaenia Horváth, 1897

Figs. 3b-g, 4 AG.

Material: Darbandikhan, 2 exx, 10.VI.1979, Rustam,

Distribuition: Iranian, previously known from Iran and Turkey.

Carpocoris coreanus Distant, 1899

Material: Many exx from Abu Ghraib, 28.III.1954, J. Mohammad; near Aqrah, 12.V.1981; near Brozah, 


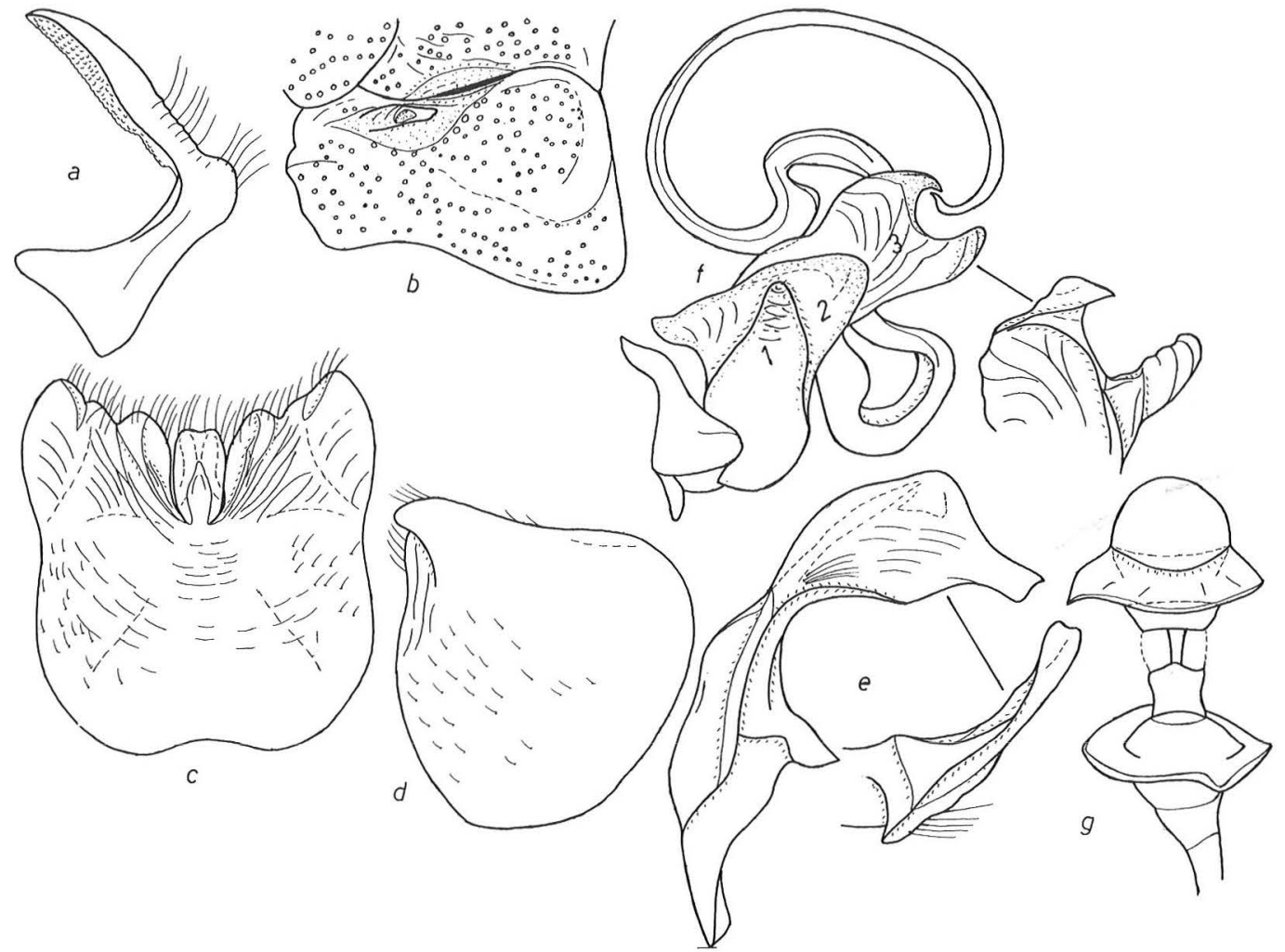

Fig. 3. Holcostethus congenitus Puchkov (ex from Khorsabad). a: style. - Agatharchus tritaenia Horvath. b: metapleuron; c-d: pygofer in ventral and in lateral view; e: style; f: aedeagus in lateral view (paired basal lobes 13 indicated with numbers); g: spermatheca.

13.V.1981; Darbandikhan, 13.V.1980; Al Hadr, 6.V.1981, Linnavuori; Kony Masy, 24.VI.1975, in AG; Ar RutbahQaim, 9.IV.1981; Al Qosh, 10.V.1981, Linnavuori; Shaqlawa, 15.VI.1976, Rustam.

Biology: On herbs in steppes.

Distribution: Irano-Turanian, spread from the Sinai Peninsula and Israel to Turkmenia; also known from Korea.

\section{Carpocoris mediterraneus mediterraneus Tamanini, 1958}

Material: Rabea, 1 ex, 22.IV.1968, F. Matti.

Distribution: Pontomediterranean; recorded from Iraq (Tamanini 1958:369).

\section{Carpocoris pudicus (Poda, 1761)}

Material: Al Qosh, 3 exx, 10.V.1981, Linnavuori; Amadia, 1 ex, 21.VI.1958, Salih; Bashika Tel Kaif, 1 ex,
24.III.1957, in AG; Top Zawe, 1 ex, 29.III.1944, H. A. Hamid.

Biology: On herbs in steppes and fields.

Distribution: Holomediterranean; recorded from Mosul, Iraq (Tamanini 1958:378).

Codophila varia (Fabricius, 1787)

Material: near Brozah, 2 exx, 13.V.1981; Sarsang, 1 ex, 26-28.VIII.1980; Sulaf, 3 exx, 27.VIII.1980, Linnavuori; Bashika, 1 ex, 24.III.1957, in AG; Kony Masy, 1 ex, 26.VI.1975, J. Abdulla; Top Zawe, 1 ex, 29.III.1944, H. Hamid, in AG.

Biology: On herbaceous plants such as Compositae and Umbelliferae in steppes and fields.

Distribution: Holomediterranean, also recorded from Iraq (Stichel 1961:754). 


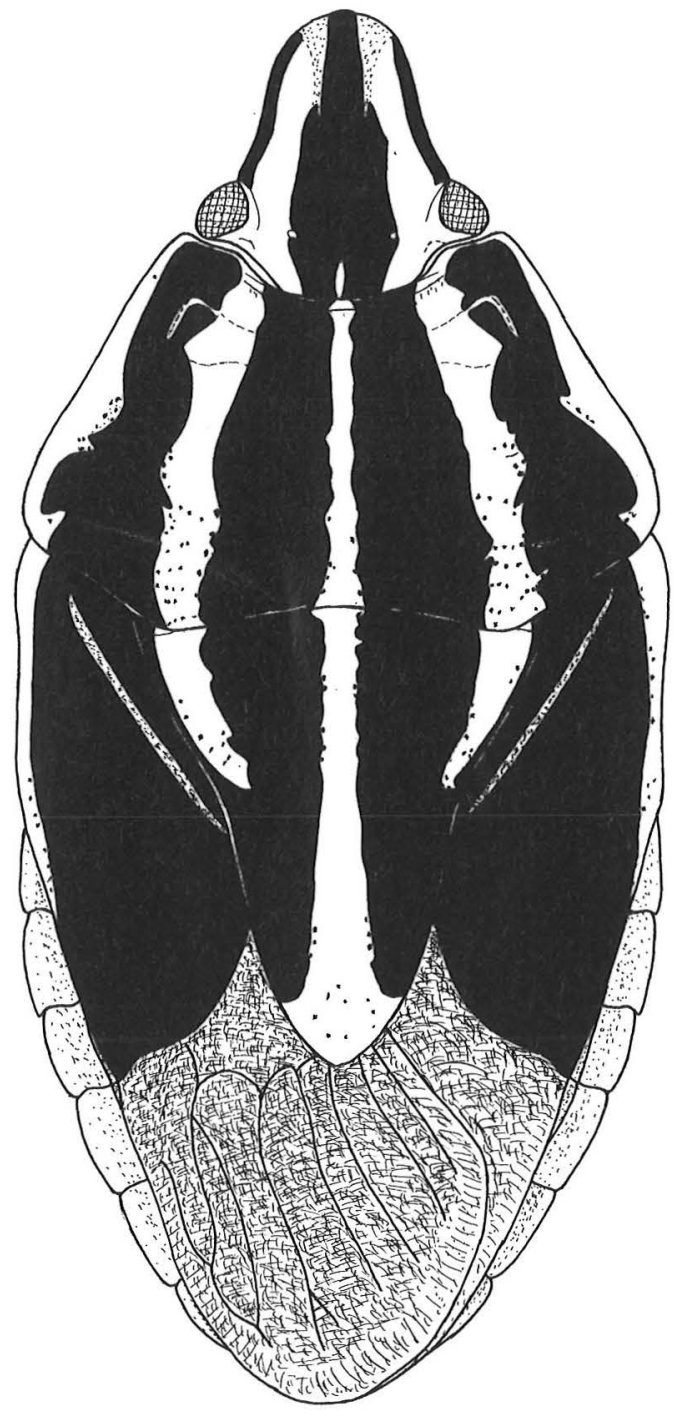

Fig. 4. Agatharchus tritaenia Horvath.

Antheminia pusio (Kolenati, 1846)

Iraq (Stichel 1961:755); Bishapur (Hoberlandt 1949:2).

Distribution: Caspian.

Antheminia lunulata (Goeze, 1778)

Material: Duhok, several exx, 26.VII.1961, M. Younis, in AG.

Distribution: Holomediterranean with a wide range in Central Europe, the Middle East and
Central Asia; recorded from Iraq (Stichel 1961: 755).

\section{Dolycoris baccarum (Linnaeus, 1758)}

Material: Numerous exx from Darbandikhan, 13.V.1981; Jebel Sinjar, 18.V.1981; Qora, 16.XI.1979; Al Qosh, 10.VI.1981; Zawita, 15.XI.1979; Shayk Addi, 11.V.1981; Sawarah Tuka, 28.VIII.1980, Linnavuori; Amadiah, 21.VI.1958, AG; Bakrajo, 28.V.1951, A. Meymarian; Basheka, 6.IV.1951, F. Younis; Deshlt Mir, 3.VI.1944, A. Meymarian; Karbala, 10.IV.1956, AG; Khorsabad, 10.VI.1984 and Korasinnik, 6.VI.1944, A. Meymarian; Mosul, 24.IV.1965, AG; Neneva, 22.IV.1955, F. Younis; Shaklawa, 30.V.1965, AG; Shura, 28.IV.1958, M. Alrawi; Top Zawe, 29.III.1944, H. Hamid; Zubeer, 23.XII.1963, AG.

Biology: On shrubs and herbs in gardens, fields and steppes.

Distribution: Euro-Siberian.

\section{Chroantha ornatula (Herrich-Schäffer, 1842)}

Material: Many exx from Anah, 10.IV.1981; Anah-Al Qaim, 9.X.1979; Basrah, 12.IV.1980; Al Hadr, 6-7.V.1981; Al Hillah, 21.IX.1979; Karbala-Ain Al Tamar, 20.X.1979; 50 km S Kirkuk, 8.XI.1979; Nasiriyah-Abu Ghar, 15.IV.1980; Nukhayb, 2.V.1980; Nukhayb-km 160; 3.V.1980; As Salman, 3.IV.1981; Sawa lake, 21.X.1979; Tharthar lake, 23.VIII.1979, Linnavuori; Abu Ghraib, 8.VII.1967, AG; Samara, 17.III.1958, M. Younis; Zubair, 8.V.1958, M. Younis.

Biology: On Chenopodiaceae such as Suaeda and Atriplex in saline habitats.

Distribution: Holomediterranean; recorded from Iraq (Stichel 1961:756).

\section{Brachynema germari (Kolenati, 1846)}

Material: Abu Ghraib, 3 exx, 11.V.1958, A. Meymarian; Anah, 1 ex, 10.IV.1981; Najaf-Shabakah, 1 ex, 23.VI.1981; Rutbah-Al Qaim, 1 ex, 4.V.1980; Tharthar lake, 3 exx, 20.X.1980, Linnavuori.

Biology: On Chenopodiaceae in saline habitats.

Distribution: Holomediterranean.

Brachynema cinctum (Fabricius, 1775)

Material: Al Kabaish, 1 ex, 27-28.VI.1980; KarbalaAin Al Tamar, 1 ex, 20.X.1979; Razaza lake, 3 exx, 7.III.1980; Sawa lake, 6 exx, 21.X.1979, 4.III and 19.IV.1980, Linnavuori. 
Biology: On Chenopodiaceae in saline habitats. Distribution: Holomediterranean.

\section{Holcogaster exilis Horváth, 1903}

Material: Sarsang, ex, 28.VIII.1980, Linnavuori.

Biology: On Juniperus oxycedrus.

Distribution: Holomediterranean.

\section{Bagrada hilaris (Burmeister, 1835)}

Material: Many exx from Anah-Al Qaim, 9.X.1979; Busayah-As Salman, 2.IV.1981; Dawrah, 15.IX.1979; Al Haditha, 13.VIII.1979; Khan Ruhabah, 6.IV.1981; NajafShabakah, 23.VI.1981; Nukhayb- km 160, 8.IV.1981, Linnavuori; Basrah, 4.II.1966, J. Al-Awi; Khan Mubawil, 1.VI.1945, A. Meymarian; Sawaira, 20.VII.1966, AG.

Biology: Very abundant on Capparis often damaging leaves.

Distribution: Sudano-Deccanian with a wide range within the Sudanese and Eremian subregions and in the Oriental region.

\section{Bagrada abeillei Puton, 1881}

Bagrada abeillei Puton 1881:121. Bagrada persica Horváth 1936:39 (Linnavuori 1986:61) Bagrada lepida Horváth 1936:41 (Linnavuori 1986:61) Bagrada cicur Horváth 1936:42 (Linnavuori 1986:61) Bagrada pallens Kiritshenko 1966:801 (Linnavuori 1986:62)

Material: Abu Ghraib, 1 ex, 1 ex, 29.IV.1961, H. Hydery, in AG; near Aqra, several exx, 29.VIII.1980; near Penjwin, 1 ex, 11.VI.1980; Al Qosh, many exx, 25.VIII.1980, 10.V.1981; near Sinjar, 1 ex, 7.V.1981, Linnavuori.

A very variable species in colouring. Genitalia illustrated in Linnavuori 1986:62-63.

Biology: In steppes in NE Iraq.

Distribution: Apparently Anatolian with a wide range into Syria, Iran and Central Asia. Recorded (as persica) from Iraq (Stichel 1961:758).

\section{Bagrada amoenula (Walker, 1870)}

Bagrada deserticola Horváth 1936:32 (Linnavuori 1964:309)

Bagrada royeri Horváth 1936:30 (Linnavuori 1964:309)

Bagrada singularis Horváth 1936:31 (Linnavuori 1964:309).

Material: Khan Ruhabah, 4 exx, 24.VI.1981, Linnavuori.
Like the preceding species very variable in colouring. Genitalia illustrated in Linnavuori 1986:62-63.

Biology: The specimens were found on Zizyphus. Generally found on desert halophytes such as Zygophyllum.

Distribution: Eastern parts of the Eremian subregion, known from Egypt, The Sudan, Yemen, and Israel.

Bagrada monticola Horváth, 1936 Fig. $5 a$

Material: Dzegamega, 1o syntype, Babadjanides, in Mus. Budapest.

Diagnosis: Resembling B. abeillei with the following differences: Body more broadly ovate and more convex. Pronotum less strongly broadening caudad, lateral margins slightly curved, humeral angles much blunter. Apex of scutellum broader. Costal margins of elytra more strongly curvate; pale basal spot on lateral margin of mesocorium very reduced.

Description: Length $5.5 \mathrm{~mm}$. Pale yellow with orangish tinge. Apex of tylus, apices of genae and base of vertex, black. Eyes reddish brown. Antennae black. Pronotum with two large black spots on disk, anterolateral angles black, anterior margin and middle of lateral margins pale yellow, rest of pronotum orangish. Scutellum orangish with large black middle spot, lateral margins subapically embrowned. Elytra black, exocorium pale ochraceous, apical transverse spot on mesocorium orange, basal pale spot very reduced, dark yellow-brown; membrane dark brown. Paratergites with distinct black spots. Under surface pale, venter with black longitudinal band on each side; parasternites orangish with triangular black spots. Legs dark yellowish brown, femora with black pattern. - Body short and broad, 1.6 $\times$ as long as broad at base of pronotum, broadly ovate. Head $1.53 \times$ as broad as long, $0.53 \times$ as broad as pronotum; vertex finely punctate, apical part of head \pm transversely wrinkled; ocular index 4.57. Proportions between antennal joints 9:13:10:15:18 (diatone 46 units). Pronotum $2.2 \times$ as broad as long in middle, lateral margins relatively weakly diverging caudad, slightly curved, humeral angles blunt; callal area strongly elevated, smooth, other parts of disk densely and 


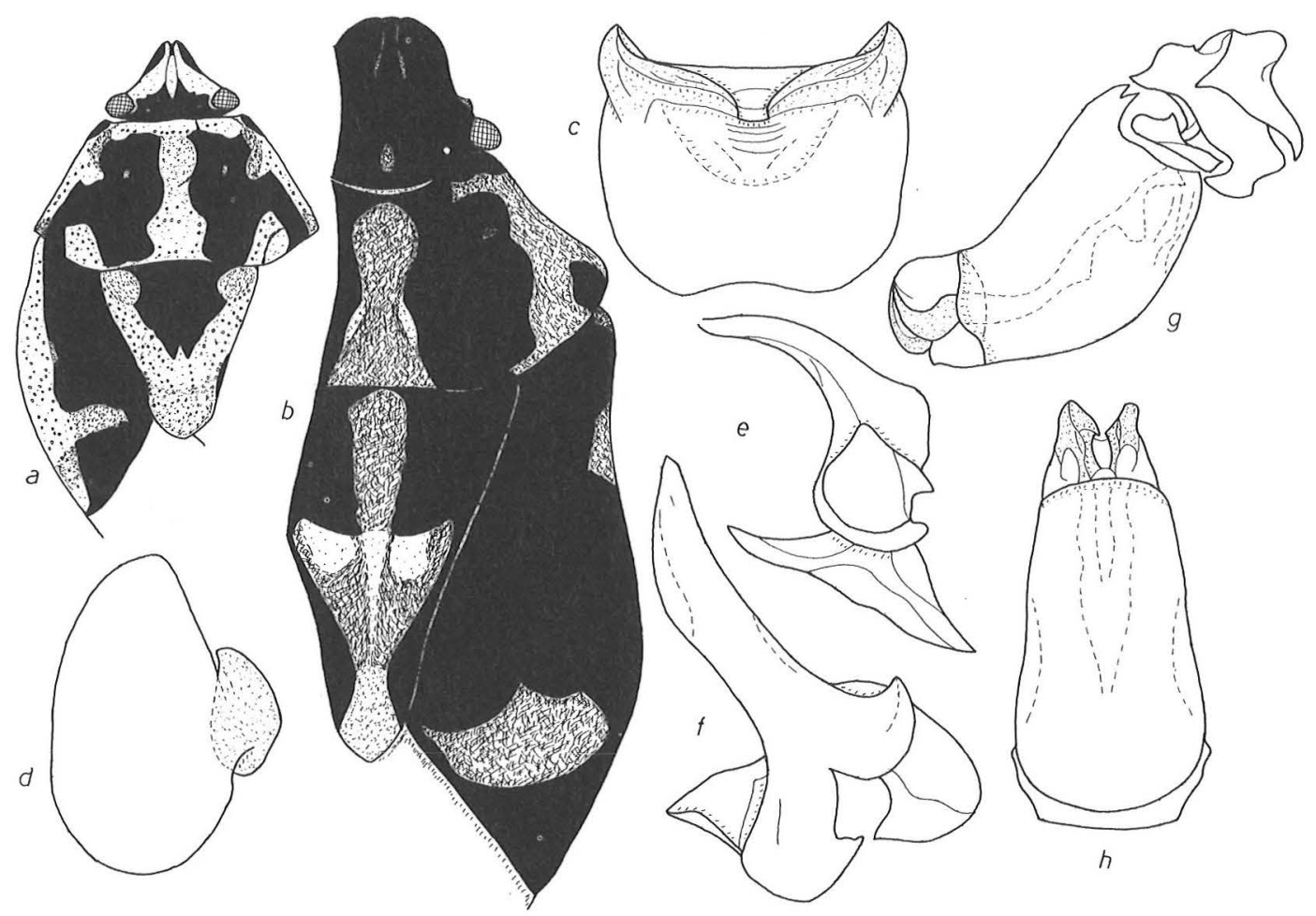

Fig. 5. Bagrada monticola Horvath. a: dorsal view. - Pseumatocoris insignis Kiritshenko: b: dorsal view; c-d: pygofer in ventral and in lateral view; e-f: style; $g-h$ : aedeagus in lateral and in ventral view.

coarsely punctate. Scutellum $1.1 \times$ as long as broad, apex remarkably broad; disk, save basal lateral callosities, coarsely and densely punctate, uneven, apex with poorly delimited callose midline. Costal margins of elytra strongly curvate, clavus and corium densely and coarsely punctate.

Distribution: Irano-Turanian; recorded from Iraq in Stichel 1961:757.

\section{Pseumatocoris insignis Kiritshenko, 1922} Figs. 5b-h

Material: Several exx from Abu Ghraib, 3.VIII.1968, J. Khalid; Kerbala, 28.V.1963, F. Matti; Kofa, 25.V.1963, M. Azher; Latifia, 27.III.1973, collector unknown; Nuamania, 24.VII.1968, M. Azher, in AG.

Male genitalia in Fig. 5c-h.

Biology: On caper, cowpea, onion and other vegetables.
Distribution: Iranian, recorded from Iraq and Iran (Stichel 1961:758).

\section{Eurydema ornatum (Linnaeus, 1758)}

Material: Many exx from Ash Shargat, 7.IV.1980; Brozah, 13.V.1981; Kuysanjag-Irbil, 12.VI.1980; Al Qosh, 10.V.1981; Penjwin, 11.VI.1980; Rashidiya, 8.VI.1981; Sinoni, 8-9.V.1981, Linnavuori; Abu Ghraib, 28.III.1950, A. Meymarian; Duhok, 21.III.1944, S. Hassan; Falluja, 22.VIII.1965, collector unknown; Hilla, 28.X.1936, Kheiri; Halabja, 20.V.1944, S. Alrawi; Khanakeen, 26.XI.1956, A. Isa; Kerbala, 4.XI.1956, Kheiri; Mosul, 15.XI.1961, S. Safar; Mussayab, 8.XII.1957, collector unknown; Qalat, 17.III.1957, M.Younis; Sowera, 3.III.1965, F. Shalabi; Top Zawi, 26.III.1944, H. Hamid.

Biology: In fields and steppes.

Distribution: Holomediterranean with a wide range into Central Europe, Central and East Asia and the Oriental region. 


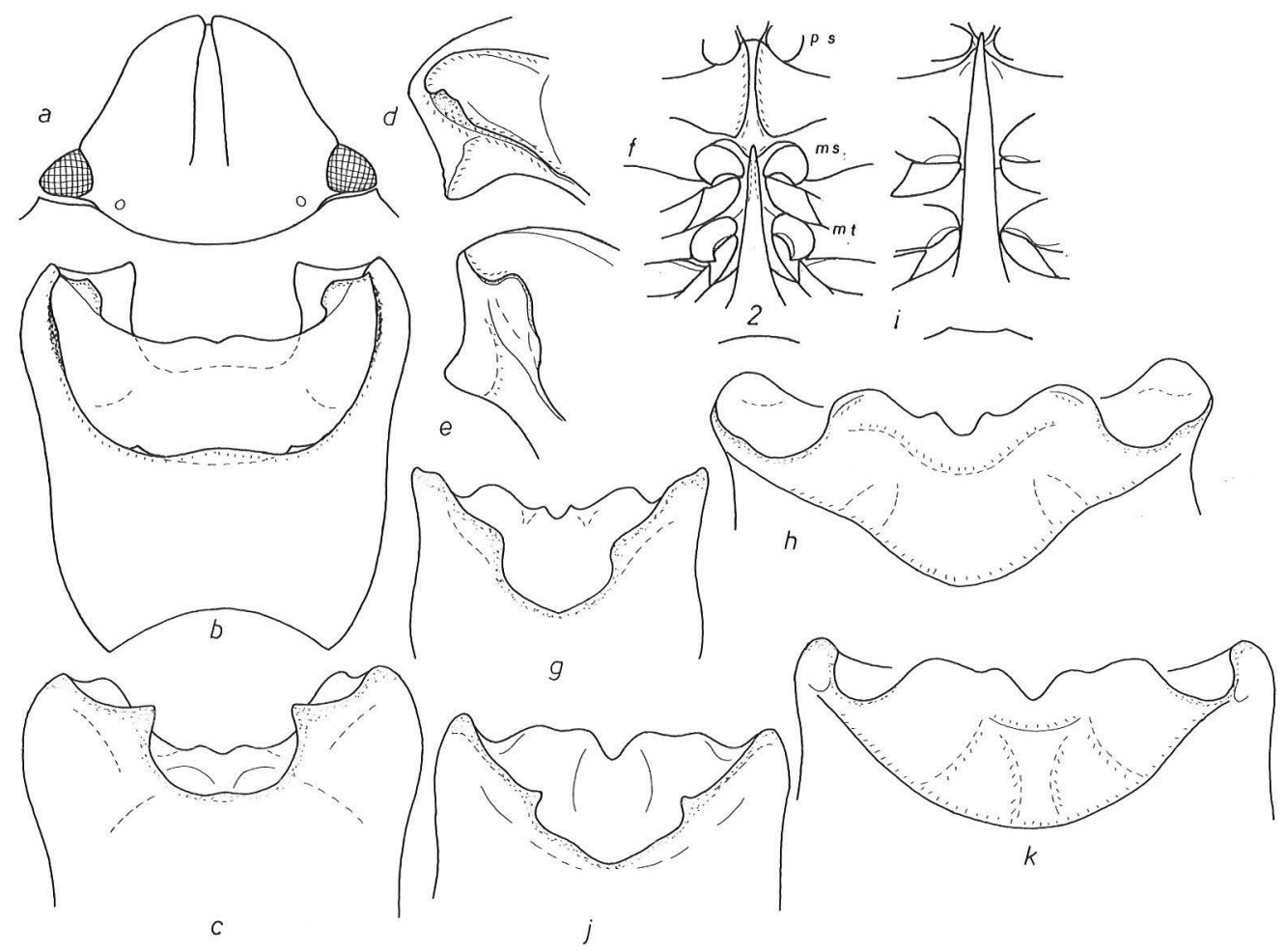

Fig. 6. Acrosternum breviceps (Jakovlev). a: head; b-c: pygofer in dorsal and in ventral view; d: caudolateral angle of pygofer in median view. - A. millierei (Mulsant \& Rey). e: caudolateral angle of pygofer in median view. - Rhaphigaster brevispina Horvath. $\mathrm{f}$ : base of venter and thorax in ventral view (ps = prosternum, $\mathrm{ms}=$ mesosternum, $\mathrm{mt}=$ metasternum 2 = 2nd sternite); $\mathrm{g}-\mathrm{h}$ : pygofer (ex from Kopet Dagh) in dorsal and in ventral view. $-R$. nebulosa (Poda). i: base of venter and thorax in ventral view; j-k: pygofer (ex from Dan, Israel) in dorsal and in ventral view.

Eurydema fieberi (Schummel, 1836)

Material: Tilgara, Arbil, 1 ex, 18.VI.1943, A. Meymarian.

Distribution: Holomediterranean.

\section{Acrosternum arabicum Wagner, 1959}

Material: Many exx from Al Qosh, 17.XI.1979; Sarsang, 26-28.VIII.1980, Linnavuori.

Biology: In steppes in northern Iraq.

Distribution: Eastern parts of the Eremian subregion, recorded from Saudi Arabia, Iran and Turkestan.
Acrosternum breviceps (Jakovlev, 1890)

Fig. 6a-d

Material: Many exx from Anah-Al Qaim, 9.X.1979; Baghdad, IV-VII.1980; Al Kabaish, 27-28.VI.1980; As Samawah, 21.X.1979; Tharthar Lake, 2.VI.1980; Linnavuori; Abu Ghraib, 12.I.1955, Dhia; Diwaniya, 11.IX.1954, S.Alyasiri; Hilla, 8.IV.1954, S.Alyasiri; Kalis, 17.VI.1974, Rustam; Mosul, 2.X.1967; A. Issa; Zakho, 21.VII.1967, M. Younis. Syria: Raqqa-Deir es Zor, 12.VIII.1979, Linnavuori.

Closely related to A. millierei (pygofer in Fig. $6 e)$ but easily distinguished by the shape of the head: broad, genae extending beyond the narrowly visible apex of tylus, and by the structure 
of the lateral lobes of the pygofer: apical margin nearly straight, median angle sharp, sclerified process on median surface narrower.

Biology: On herbs in saline habitats; at lamps.

Distribution: Irano-Turanian, recorded from Turkestan, Iraq and Saudi-Arabia (Stichel 1961:763).

Acrosternum millierei (Mulsant \& Rey, 1866)

Recorded from Iraq (Stichel 1961:763).

Distribution: Holomediterranean with a wide range in the Middle East and the Ethiopian region.

\section{Nezara viridula (Linnaeus, 1758)}

Material: Numerous exx from Abu Ghraib, 26.VII. 1961, Haidari; Baghdad, 2.VII.1946, collector unknown; Balad, 7.III.1958, Younis; Hilla, 28.X.1956, S. Ibrahim; Kut, 7.III.1950, Fathy; Ramadi, 9.III.1958, Younis; Rashidiyah, 8.VI.1981, Linnavuori; Suwaira, 10.XI.1966, J. Alani.

Biology: In fields on cotton, broad bean and other herbs.

Distribution: Cosmopolitan, except for cold regions. Recorded from Iraq (Stichel 1961:763).

\section{Piezodorus lituratus (Fabricius, 1794)}

Material: Several exx from Anab, 19.III.1944, Yousuf; Bakraja, 26.V.1951, A. Meymarian; Begbagi, 9.III.1944, S. Hassan; Biyara, 19.III.1944, M. Yousuf; Falaja, 21.XI.1957, collector unknown; Kani Khesman, 10.III.1944, S. Hassan; Sulaymaniya, 14.IV.1950, collector unknown; Top Zawe, 29.III.1944, H. Hamid, in AG.

Distribution: Holomediterranean with a wide range into Central Europe and the Middle East.

\section{Pausias martini (Puton, 1890)}

Material: Dukan, 1 ex, 12.VI.1980, Linnavuori; Shaqlawa, 2 exx, 27.II.1967, S. Safar, in AG.

Biology: At Dukan found on Quercus.

Distribution: Recorded from Syria, Iraq, Armenia, Caucasia, and Iran (Stichel 1961:765).
Anchesmus rubriplaga (Walker, 1867)

Material: Several exx from Hilla, 21.IX.1979; As Salman, 19.IV.1980, Linnavuori.

Biology: On Tamarix.

Distribution: Eremian.

Rhaphigaster nebulosa (Poda, 1761)

Fig. 6i-k

Material: Sulaf, 3 exx, 27.VIII.1973, Linnavuori.

The variability of $R$. nebulosa was treated in Linnavuori 1960:19-21. Specimens from the Pontomediterranean area differ from Central and West European ones in the more elongate body and somewhat paler colouring. Moreover, the head is \pm more elongate and the antennae are somewhat longer. Both forms are connected by intermediates, however. Specimens from Israel were incorrectly regarded as $R$. brevispina Horváth, 1889 by me. The genuine $R$. brevispina, an Irano-Turanian species, is readily distinguished by the short ventral spine which extends only to the base of the mesosternum (Fig. 6f) (to the base of the prosternum in nebulosa). Moreover the shape of the pygofer (Fig. $6 \mathrm{~g}-\mathrm{h}$ ) is somewhat different.

Biology: On broad-leaved trees in a grove in northern Iraq.

Distribution: Holomediterranean, extending into Central Europe, the Middle East and Central Asia. Recorded from Iraq (Stichel 1961:766).

\section{Asopinae}

Andrallus spinidens (Fabricius, 1878)

Material: Abu Ghraib, 1 ex, 23.I.1955, Y. Fattah; Karbala, 1 ex, 4.XI.1956, W. Muneer.

Biology: At Karbala found on cucumber.

Distribution: Tropicopolitan. Recorded from Iraq (Stichel 1961:768).

\section{Zicrona caerulea (Linnaeus, 1758)}

Material: Baghdad, 3 exx, 23.IX.1979, Linnavuori; Bakraja, many exx, 7.XI.1950, A. Meymarian.

Biology: In moist habitats in gardens.

Distribution: Holarctic. 


\section{Stenocephalidae}

Dicranocephalus setulosus (Frey-Gessner, 1874)

Material: Sarsang, 1 ex, 26-28.VIII.1980, Linnavuori.

Biology: On Euphorbia like the other species of the genus.

Distribution: Holomediterranean.

\section{Dicranocephalus albipes (Fabricius, 1781)}

Material: near Penjwin, 2 exx, 11.VI.1980, Linnavuori.

Distribution: Holomediterranean, recorded from Iraq (Stichel 1961:708).

\section{Dicranocephalus marginatus (Frey-Gessner, 1874)}

Material: Abu Ghraib, 3 exx, 17-22.VI.1968, J. Alani; Jebel Sinjar, 1 ex, 8.V.1980; Mosul, 1 ex, 28.VIII.1980; Sarsang, 4 exx, 26-28.VIII.1980, Linnavuori.

Distribution: Irano-Turanian, extending from Syria to Turkestan; recorded from Baghdad (Lansbury 1965:68).

\section{Dicranocephalus bianchii (Jakovlev, 1901)}

Material: Anah-Al Qaim, 1 ex, 9.X.1979; Nukhayb, 1 ex, 2.V.1980; Ukhaydir-Nukhayb, 3 exx, 2.V.1980, Linnavuori.

Distribution: Eastern parts of the Eremian subregion, extending from Egypt (the Sinai Peninsula) to Iran.

\section{Dicranocephalus pallidus (Signoret, 1879)}

Material: Abu Ghraib, 1 ex, 14.V.1955 and Qurna, 1 ex, 25.VIII.1966, collector unknown in AG; Amarah, 1 ex, 8.VIII.1964, F.Al-Saizani; As Salman, 1 ex, 18.IV.1980; Baghdad, 1 ex, VIII.1980; Nukhayb, 1 ex, 2.V.1980; Ukhaydir-Nukhayb, 1 ex, 2.V.1980, Linnavuori.

Distribution: Eremian.

\section{Coreidae}

\section{Coreinae}

Omanocoris versicolor (Herrich-Schäffer, 1841)

Material: Baghdad, 3 exx, 23.IX.1979, Linnavuori; numerous exx in AG from Abu Ghraib, 1-16.V.1943, 5.XI.1966, 1.VII.1967, A.Meymarian and I.Khalid; Sowera, 31.III.1955, F.Shalabi.
Biology: On Leguminosae. I collected it in numbers from an unidentified Leguminosae tree at Tarut in Saudi Arabia. The AG material was collected from string-bean and cotton.

Distribution: Known from the Oriental Region, Iran, Iraq (Nasiriya, China 1934:429), and Saudi Arabia.

\section{Gonocerus acuteangulatus (Goeze, 1778)}

Material: Sarsang, 1 ex, 27.VIII.1980; Sulaf, 1 ex, 27.VIII.1980, Linnavuori; Twela, 4 exx, 12.VII.1975, Rustam.

The Iraqi specimens studied have acute humeral angles of the pronotum and represent the form acutangula Puton, 1881.

Biology: On Juniperus oxycedrus.

Distribution: Holomediterranean with a wide range in Central Europe.

\section{Gonocerus juniperi Herrich-Schäffer, 1839}

Material: Sarsang, 3 exx, 26-27.VIII.1980, Linnavuori.

Biology: On Juniperus oxycedrus.

Distribution: Holomediterranean, with a wide range in Central Europe.

\section{Coreus marginatus syriacus Blöte, 1935}

Material: Barzan, 2 exx, 29.VIII.1980; Karadag, 2.VI.1980; Sulave, 2 exx, 2.VII.1975, Rustam; Zibar, 1 ex, 14.IV.1953, Mahmood.

Distribution: The nominate form C. marginatus marginatus (Linnaeus, 1787) Euro-Siberian, ssp. syriacus known from Israel, Syria and Iraq (Shaqlawa, Hoberlandt 1953:378).

\section{Syromastus rhombeus (Linnaeus, 1767)}

Recorded from Iraq (Stichel 1961:712).

Distribution: European, extending to Iran and Turkestan.

\section{Enoplops disciger (Kolenati, 1845)}

Recorded from Iraq (Stichel 1961:712).

Distribution: Pontomediterranean.

\section{Centrocoris spiniger (Fabricius, 1781)}

Material: Several exx from Aqra, 29.VIII.1980, 12.V.1981; Dukan, 12.VI.1980; Al Qosh, 10.V.1981; Shayk Addi, 11.V.1981; Sulaf, 27.VIII.1980, Linnavuori. 
Biology: In steppes of northern Iraq.

Distribution: Holomediterranean.

Centrocoris volxemi (Puton, 1878)

Material: Abu Ghraib, 1 ex, 30.XII.1943, R. Meymarian; Hilla, 1 ex, 21.IX.1979; Rutba-Al Qaim, 1 ex, 5.V.1980, Linnavuori.

Biology: In semideserts of central Iraq.

Distribution: Irano-Turanian.

Cercinthus lehmanni (Kolenati, 1856)

Material: Razaza lake, 1 ex, 24.VI.1981; Tharthar lake, 1 ex, 6.VI.1981, Linnavuori.

Biology: In desert habitats.

Distribution: Eremian, extending from the Canary Islands to Turkestan.

\section{Spathocera lobata (Herrich-Schäffer, 1840)}

Material: Abu Ghraib, 1 ex, 15.III.1975, Latifia, 1 ex, 27.III.1973, collector unknown, in AG.

Distribution: Holomediterranean.

Prionotylus brevicornis (Mulsant \& Rey, 1852)

Material: Darbandikhan, 1 ex, 13.V.1980; Sawarah Tuka, 1 ex, 28.VIII.1980; Zawita, 1 ex, 26.VIII.1980, Linnavuori.

Biology: On grasses in steppes.

Distribution: Holomediterranean.

\section{Phyllomorpha lacerata Herrich-Schäffer, 1835}

Material: Aqra, 1 ex, 29.VIII.1980, Linnavuori; Zakho, 2 exx, 25.VI.1941, Younis.

Distribution: Pontomediterranean.

\section{Pseudophloeinae}

Arenocoris intermedius (Jakovlev, 1883)

Material: Khan Ruhabah, 1 ex, 24.VI.1981; NajafShabakah, 1 ex, 23.VI.1981; Safwan, 1 ex, 13.IV.1980, Linnavuori.

Biology: On ground among plants in semideserts and steppes.

Distribution: Holomediterranean.
Arenocoris waltli (Herrich-Schäffer, 1834)

Material: Jebel Sinjar, 1 ex, 8.V.1981, Linnavuori.

Biology: Under plants in a hilly steppe.

Distribution: Holomediterranean.

Ceraleptus obtusus (Brullé, 1839)

Recorded from Baghdad (China 1938:429).

Distribution: Holomediterranean.

Coriomeris vitticollis Reuter, 1900

Material: Abu Ghraib, 1 ex, 22.VII.1954, collector unknown, in AG; Al Qosh, 1 ex, 17.XI.1979, Linnavuori.

Distribution: Apparently Irano-Turanian. Recorded from Iraq (Shaqlawa, Hoberlandt 1953:378).

Coriomeris affinis (Herrich-Schäffer, 1839)

Material: Sawarah Tuka, several exx, 28.VIII.1980, Linnavuori.

Distribution: Holomediterranean.

Strobilotoma typhaecornis (Fabricius, 1803)

Material: Baquba, 1 ex, 30.III.1958, A.Yasiki; Khalis, 2 exx, 30.III.1958, M.Arawi; Al Qosh, 1 ex, 10.V.1981, Linnavuori; Sulaymaniya, 1 ex, 11.V.1957, Abid.

Distribution: Holomediterranean; recorded from Iraq (Stichel 1961:716).

\section{Alydidae}

\section{Camptopus lateralis (Germar, 1817)}

Material: Several exx from Aqra, 29.VIII.1980; Darbandikhan, 13.V.1980; Dawrah, 15.IX.1979; Al Qosh, 10.VI.1981; Salahuddin, 13-14.VI.1980; Shayk Addy, 11.V.1981; Sulaf, 27.VIII.1980, Linnavuori; Rabeah, 2 exx, 10.IV.1969, collector unknown, in AG.

Biology: On grasses and other herbs in steppes.

Distribution: Holomediterranean, extending into Central Europe and Turkestan and Afghanistan. 
Camptopus illustris Horváth, 1899

Material: Shaqlawa, 1 ex, 11.VII.1976, Rustam.

Distribution: Known from Turkey, Caucasia, Iraq, and Iran (Stichel 1961:717).

\section{Riptortus linearis (Fabricius, 1775)}

Material: Several exx from Abu Ghraib, 10.IV.1954, 18.VIII.1958, A.Haidari, S.Rasheed; Baghdad, 23.IX.1979, Linnavuori; Basra, 21.III.1951, Younis; Kalis, 17.VI.1979, Rustam; Kefil, 23.III.1975, collector unknown, in AG; Kirkuk, 31.VII.1967, Younis; Rashidiyah, 8.VI.1981, Linnavuori; Samara, 14.VI.1957, Younis; Zafaraniyah, 21.IX.1942, A.Meymarian.

Biology: On undergrowth in gardens.

Distribution: The Oriental Region, extending to China and Iran.

Nariscus longirostris Linnavuori, 1974

Material: Kirkuk (Haweja), 1 ex, 1.VIII.1961, J.Alani.

Description in Linnavuori 1974:170-171 and 1987:25.

Distribution: Otherwise known only from Israel.

\section{Rhopalidae}

Nomenclature as in Puchkov 1986.

\section{Rhopalinae}

\section{Corizus hyoscyami (Linnaeus, 1758)}

Material: Brozah, 1 ex, 13.V.1981; Darbandikhan, 3 exx, 13.V.1980; Qora, 1 ex, 16.XI.1979; Al Qosh, 1 ex, 12.V.1981; Sawarah Tuka, 1 ex, 28.VIII.1980; Shaykh Addi, 1 ex, 11.V.1981, Linnavuori; Kani Khesman, 1 ex, 10.III.1944, S.Hassan; Rabeah, 3 exx, 10.IV.1969, collector unknown, in AG.

Biology: In steppes and hilly habitats in northern Iraq.

Distribution: Euro-Siberian, extending to China and North Africa.

\section{Corizus fenestella fenestella Horváth, 1917}

Material: Al Qosh, 1 ex, 17.XI.1979; Zawita, 1 ex, 26.VIII.1980, Linnavuori.
Biology: In hilly steppes of northern Iraq.

Distribution: Known from Turkey, Caucasia and Iran.

Brachycarenus tigrinus (Schilling, 1829)

Material: Darbandikhan, 1 ex, 13.V.1980; Sinoni, 1 ex, 8-9.V.1981; Zawita, 1 ex, 26.VIII.1980; Linnavuori.

Biology: In hilly steppes of northern Iraq.

Distribution: European, extending to Mongolia and China.

\section{Liorhyssus hyalinus (Fabricius, 1794)}

Material: Numerous exx from Abu Ghar, 16.IV.1980; Abu Ghar-Busayah, 16.IV.1980, Linnavuori; Abu Ghraib, 7.V.1953, A.Isa; Anah-Al Qaim, 5.V.1980; Baghdad, IX.1979, VIII.1980; Al Fallujah, 5.X.1979; Al Hadr, 67.V.1981; Halabja, 11.VI.1980; Jebel Sinjar, 8.V.1981, Linnavuori; Karbala, 9.XI.1956, S.Alyasiri; Kufa, 5.XI.1956, S.Ibrahim; Karbala-Ain Al Tamar, 20.X.1979, Linnavuori; Mosul, 31.X.1968, J.Abdulla; Nasiriyah-Abu Ghar, 15.IV.1980; Nukhayb- km 160, 9.V.1980; Penjwin, 11.VI.1980; Qora, 13.VI.1980; Al Qosh, 10.V.1981, Linnavuori; Rabea, 22.IV.1968, F.Matty; Ramadi, 21.VI.1966, in AG; Rashidiyah, 8.VI.1981; Rawah-Al Hadr, 6.V.1981; Razaza lake, 6.VI.1981; Safwan, 13.IV.1980; As Salman, 3.IV.1981; Sawarah Tuka, 28.VIII.1980; Shaykh Addi, 11.V.1981, Linnavuori; Shora, 30.IX.1956, S.Alyasiri; Sinoni, 8-9.V.1981; Tharthar lake, 23.VIII.1979, Linnavuori; Tikrit, 7.IV.1969, J.Alani; Ukhaydir-Nukhayb, 2.V.1980; Waqisah, 1.V.1980; Az Zubayr, 14.IV.1980, Linnavuori, Kuwait: Al Jahra, 17.III.1981, Linnavuori.

Biology: Common in fields, gardens, steppes, and semideserts.

Distribution: Cosmopolitan, except for cold areas.

Rhopalus (Aeschyntelus) maculatus (Fieber, 1837)

Material: Sulaf, 1 ex, 27.VIII.1980, Linnavuori.

Distribution: Euro-Siberian; recorded from Shaqlawa (Hoberlandt 1953:380).

Rhopalus (s. str.) subrufus (Gmelin, 1790)

Material: Zawita, 1 ex, 20.VIII.1980, Linnavuori.

Euro-Siberian.

\section{Stictopleurus abutilon pictus (Fieber, 1861)}

Material: Several exx from Aqra, 12.V.1981; Ash Shargat, 7.IV.1980; Al Hadr, 6.V.1981; Penjwin, 
11.VI.1980; Al Qosh, 10.V.1981; Khorsabad, 11.V.1981; Sawarah Tuka, 28.V.1980, Linnavuori.

Biology: In steppes of northern Iraq. Distribution: Holomediterranean.

\section{Stictopleurus riveti Royer, 1923}

Material: Several exx from Abu Ghraib, 11.IX.1966, in AG; Baghdad, V-VI.1981; Al Hadr, 6-7.V.1981; RutbaQaim, 8-9.III.1980; As Salman-Shabakah, 4.IV.1981; Sarsang, 26-28.VIII.1980; Waqisah, 1.V.1980, Linnavuori.

Biology: In steppes and semideserts.

Distribution: Holomediterranean.

Stictopleurus ribauti Vidal, 1952

Distribution: Eremian; recorded from Iraq (Göllner-Scheiding 1975:41).

\section{Maccevethus errans caucasicus (Kolenati, 1845)}

Material: Aqra, 1 ex, 12.V.1981; Jebel Sinjar, 3 exx, 8.V.1981; Al Qosh, 2 exx, 10.V.1981; Sarsang, 2728.VIII.1980; Sinjar, 1 ex, 7.V.1981, Linnavuori; Erbil, 1 ex, 30.V.1969, J.Mohmed; Grmawe, 1 ex, 8.IV.1944, S. Hassan; Nenewa, 1 ex, 10.XI.1965, in AG; Shaqlawa, 2 exx, 11.VII.1976, Rustam.

Biology: In steppes of northern Iraq.

Distribution: Holomediterranean.

Maccavethus corsicus persicus Jakovlev, 1882

Distribution: Pontomediterranean; recorded from Shaqlawa (Hoberlandt 1953:378).

\section{Chorosomatini}

Agraphopus (Leptoceraea) viridis (Jakovlev, 1873)

Agraphopus femoralis Horváth, 1897 (Puchkov 1986:99)

Material: Many exx from Baghdad, IV-VII.1980; Basra, 25-29.VI.1980; Ain Al Tamar, 17.VII.1980; Al Hillah, 21.IX.1980; A1 Kabaish, 27-28.VI.1980; NasiriyaAbu Ghar, 15.IV.1980; Tharthar lake, 5.X.1979, 2.VI.1980; Linnavuori.

Biology: In moist habitats in gardens, fields and shores.

Distribution: Pontomediterranean.
Agraphopus (Agraphopus) lethierryi Stål, 1872

Material: Ana, 2 exx, 8.X.1979; Aqra, 1 ex, 29.VIII. 1980; Baghdad, several exx, IV-VIII.1980; Muhammadi, 2 exx, 8.X.1979, Linnavuori.

Biology: On grasses in fields and shores.

Distribution: Holomediterranean with a wide range in the Ethiopian Region.

Agraphopus (Agraphopus) suturalis Reuter, 1900

Material: Baghdad, several exx, IV-VII.1980, Linnavuori.

Biology: On Panicum turgidum and other desert grasses.

Distribution: Eremian.

Chorosoma schillingi (Schilling, 1829)

Material: Several exx from Aqra, 12.V.1981; Brozah, 13.V.1981; Al Hadr, 6.V.1981; Al Qosh, 10.V.1981; Zawita, 15.XI.1979, Linnavuori. Iraq.

Biology: On grasses in steppes of northern

Distribution: European.

\section{Pyrrhocoridae}

\section{Pyrrhocoris apterus (Linnaeus, 1758)}

Material: Several exx from Baghdad, IV-V.1980, Linnavuori; Abu Ghraib, 20.V.1945, H.Hamid; Khanakin, 13.XII.1957, in AG; Neneva, 14.XI.1965, in AG; Shaqlawa, 11.VII.1976, in AG; Sulaymaniyah, 27.I.1968, Ronak; Zakho, 24.IX.1954, R.Ali.

Biology: On ground under plants in gardens and fields.

Distribution: Holarctic.

\section{Scantius aegyptius (Linnaeus, 1758)}

Material: Numerous exx from Busaya, 2.IV.1981; Nukhaib-Ratawi, 9.IV.1981; Al Qosh, 25.VIII.1980; As Salman, 3.IV.1981; Waqisah, 1.V.1980; Linnavuori; Abu Ghraib, 3.II.1955, R.Ali; Diyala, 10.XI.1954, in AG; Rutba, 8.VI.1966, in AG; Samara, 7.III.1958, Younis; Tikrit, 16.III.1958, Alyasiri. Kuwait: Al Wafra, 17.III.1981, Linnavuori.

Biology: Under plants in gardens and fields.

Distribution: Holomediterranean. 


\section{Scantius forsteri (Fabricius, 1781)}

Material: Zubeer, 4 exx, 8.V.1969, M. Younis. Kuwait: Al Jahra, 1 ex, 17.III.1981, Linnavuori.

Biology: Under plants in dry habitats.

Distribution: Ethiopian; also known from Sicily, Egypt, Saudi Arabia, Israel, Iran, and India.

\section{References}

China, W. E. 1938: Hemiptera from Iraq, Iran and Arabia. — Field Mus. Nat. Hist. Zool., Ser. 20:427-437. Chicago.

Göllner-Scheiding, U. 1986: Revision der Gattung Odontoscelis Laporte de Castelnau 1832 (Heteroptera, Scutelleridae). — Deutsche Entomol. Zeit., N.F. 33:95-127.

Hoberlandt, L. 1944: Some Hemiptera-Heteroptera collected in North East Iraq. - Acta Entomol. Mus. Nat. Pragae 26:1-9.

- 1953: Hemipteren-Heteropteren von Shaqlawa in südKurdistan. Beitr. zur Entomol. 3:377-384.

Horváth, G. 1897: Description d'Hémiptères nouveaux et notes diverses. - Rev. Entomol. 1897:81-97.

- 1936: Monographia Pentatomidarum generis Bagrada. - Ann. Hist.-Nat. Mus. Nat. Hungarici 30:22-47.

Jakovlev, V. Е. (Яковлев, В. Е.) 1873: [Materials for the insect fauna of the European part of Russia III.] (In Russian) - Trud. Rus. Entomol. 1874. T.7 (2):30-41.

Josifov, M. 1969: Über die Taxonomie von Ventocoris (Selenodera) falcatus (Cyrillus, 1787) (Heteroptera, Pentatomidae). - Acta Entomol. Mus. Nat. Pragae 38:67-72.

Kerzhner, I. М. (Керзнер, И. М.) 1976: [New or little known Heteroptera from Mongolia and from adjacent regions of the USSR.] (In Russian) - Nasek. Mongol. 4:30-86.

Kiritshenko, А. N. (Кириченко, А. Н.) 1966: [HemipteraHeteroptera collected by D.M. Steinberg in Iran 1955.] (in Russian) - Entomol. Obozr. 45:798-805.

Klug, J. C. F. 1845: Symbolae physicae seu icones \& descriptiones insectorum 5: 10 Tables.

Lansbury, I. 1965-1966: A revision of the Stenocephalidae Dallas 1852 (Hemiptera-Heteroptera). — Entomol. Monthly Mag. 101:52-92, 145-160.

Linnavuori, R. E. 1964: Hemiptera of Egypt, with remarks on some species of the adjacent Eremian region. Ann. Zool. Fennici 1:306-356.
- 1965: Additions to the Hemipterous fauna of Israel. Ann. Entomol. Fennici 31:237-241.

- 1974: Hemipterological studies. - Ann. Entomol. Fennici 40:167-171.

- 1984: New species of Hemiptera Heteroptera from Iraq and the adjacent countries. - Acta Entomol. Fennici 44:1-59.

- 1987: Alydidae, Stenocephalidae and Rhopalidae from West and Central Africa. - Acta Entomol. Fennici 49:1-36.

- 1986: Heteroptera of Saudi Arabia. — Fauna of Saudi Arabia 8:31-197.

- 1993: Cydnidae of West, Central and North-East Africa. - Acta Zool. Fennica 192. 148 pp.

Puchkov, V. G. (Пучков, В. Г.) 1965: [Shieldbugs of Middle Asia (Hemiptera, Pentatomoidea.)] (In Russian) — Acad. Nauk Kirgizskoi SSR Inst. Biol. 1965:1329.

- 1986: [Rhopalid bug fauna of the USSR.] (In Russian) - Acad. Nauk. SSSR. Zool. Inst. 146:1-132.

Puton, A. 1881: Enumération des Hémiptéres recoltés en Syrie par M. Abeille de Perrier avec la description des espéces nouvelles. - Mitteil. Schweiz. Entomol. Ges. 6:121.

Reuter, O. M. 1900: Heteroptera palaearctica nova et minus cognita descripsit I. — Öfv. Finska Vetensk.-Soc. Förh. 42:209-239.

Seidenstücker, G. 1964: Zur Systematik von Bledionotus, Bethylimorphus und Thaumastella Horváth (Heteroptera, Lygaeidae). - Reichenbachia 3:269-279.

- 1975: Über anatolische Schildwanzen (Heteroptera, Pentatomidae). - Reichenbachia 15:259-268.

Signoret, V. 1881: Groupe des Cydnides 4. - Ann. Soc. Entomol. France 1881:423-436.

Stichel, W. 1961: Illustrierte Bestimmungstabellen der Wanzen II. Europa. 4(23-24):705-768.

- 1962: Illustrierte Bestimmungstabellen der Wanzen II. Europa. 4(25):769-800.

Tamanini, L. 1958: Revisione del genere Carpocoris Klt. con speciale riguardo alle specie italiane (Hemiptera, Heter., Pentatomidae). - Mem. Mus. Civ. St. Nat. 6:333-388.

Wagner, E. 1965: Die taxonomische Bedeutung des Baues der Genitalien des Männchens bei der Gattung Sciocoris Fallen 1829 (Hem. Het. Pentatomidae). Acta Entomol. Mus. Nat. Pragae 36:91-167.

Walker, F. 1867: Catalogue of the specimens of Hemiptera Heteroptera in the collection of the British Museum. 1. — London. 240 pp.

Received 12.XII.1991 